Please do not remove this page

RMIT

UNIVERSITY

\title{
Optically Stimulated Artificial Synapse Based on Layered Black Phosphorus
}

Ahmed, Taimur; Kuriakose, Sruthi; Mayes, Edwin; Ramanathan, Rajesh; Bansal, Vipul; Bhaskaran, Madhu; Sriram, Sharath

https://researchrepository.rmit.edu.au/esploro/outputs/9921861148801341/filesAndLinks?institution=61RMIT_INST\&index=null

Ahmed, T., Kuriakose, S., Mayes, E., Ramanathan, R., Bansal, V., Bhaskaran, M., Sriram, S., \& Walia, S. (2019). Optically Stimulated Artificial Synapse Based on Layered Black Phosphorus. Small, 15(22), 1-1. https://doi.org/10.1002/smll.201900966

Document Version: Accepted Manuscript

Published Version: https://doi.org/10.1002/smll.201900966

Repository homepage: https://researchrepository.rmit.edu.au

(c) 2019 WILEY-VCH Verlag GmbH \& Co. KGaA, Weinheim

Downloaded On 2023/04/26 11:33:43 +1000

Please do not remove this page 


\section{Optically stimulated artificial synapse based on a layered elemental material}

Taimur Ahmed ${ }^{1, *}$, Sruthi Kuriakose ${ }^{1}$, Edwin L.H. Mayes ${ }^{2}$, Rajesh Ramanathan ${ }^{3}$, Vipul Bansal ${ }^{3}$, Madhu Bhaskaran ${ }^{1}$, Sharath Sriram ${ }^{1}$ and Sumeet Walia ${ }^{1, *}$

${ }^{1}$ Functional Materials and Microsystems Research Group and the Micro Nano Research Facility, RMIT University, Melbourne VIC 3001, Australia

${ }^{2}$ RMIT Microscopy and Microanalysis Facility, RMIT University, Melbourne, VIC 3001, Australia.

${ }^{3}$ Sir Ian Potter NanoBioSensing Facility and NanoBiotechnology Research Laboratory, School of Science, RMIT University, Melbourne VIC 3001, Australia

*Correspondence to: taimurahmad1@gmail.com, sumeet.walia@rmit.edu.au

Keywords: artificial synapse, neuromorphic computation, black phosphorus, photonic signal processing, bio-inspired electronics, short-term plasticity, long-term plasticity

The translation of biological synapses onto a hardware platform is an important step toward the realization of brain-inspired electronics. However, to mimic the biological synapses, devices till-date continue to rely on the need for simultaneously altering the polarity of an applied electric field or the output of these devices is a photonic synapse, instead of an electrical synapse. As the next big step towards practical realization of optogenetics inspired circuits that exhibit fidelity and flexibility of biological synapses, optically-stimulated synaptic devices without a need to apply polarity-altering electric field are needed. Arising from the observation of a unique photoresponse in black phosphorus (BP), here we report an all-optical pathway to emulate excitatory and inhibitory action potentials by exploiting oxidation-related defects. These optical synapses are capable of imitating key neural functions such as psychological learning and forgetting, spatiotemporally correlated dynamic logic and Hebbian spike-time dependent plasticity. The functionality is also demonstrated on a flexible platform suitable for wearable electronics. These 
low-power consuming devices are highly attractive for deployment in neuromorphic architectures. The manifestation of cognition and spatiotemporal processing solely through optical stimuli provides an incredibly simple and powerful platform to emulate sophisticated neural functionalities such as associative sensory data processing and decision making.

\section{INTRODUCTION}

Realization of neuromorphic systems is recognized as a viable technological route to achieve brain-like computational capabilities in artificial networks. These neuromorphic systems have been efficient in imitating the biological neural networks to solve complex tasks such as learning, multi-object detection, classification and recognition. ${ }^{[1-4]}$ In state-of-the-art neuromorphic systems, synaptic plasticity - a fundamental computational function of a nervous system to execute cognitive functions - has been mimicked by several types of artificial synapses based on transistors ${ }^{[5-7]}$ and memristors. ${ }^{[1,8-10]}$ Although these ingenious neuromorphic systems have been developed through mature technologies, there are limitations to achieve the processing efficiency of a human brain. ${ }^{[11]}$ For instance, unlike neuromorphic systems, biological neural systems exhibit associative learning via sensory (olfactory, ocular, somatosensory etc.)

stimuli. ${ }^{[12,13]}$ As such, neuromorphic sensors imitating biological architecture of sensory organs result in hardware redundancy, power consumption and computational latency. ${ }^{[14]}$ Furthermore, artificial neural networks utilize a series of electrical signals and interconnections to emulate synaptic action potentials which limit the processing speed due to the bandwidth connectiondensity trade-off. ${ }^{[15]}$ To overcome these limitations, innovative architectures and exotic functional elements are required to reach closer to the computational capabilities (such as associative learning and decision making) of the biological neural systems. 
The application of optical stimuli to implement a neuromorphic system renders ultrafast computational speed due to high bandwidth, low crosstalk and ultralow power-consumption. ${ }^{[15-}$ ${ }^{17]}$ Development of optically and electrically stimulated synaptic devices based on one- and two-dimensional (2D) materials such as carbon nanotubes, ${ }^{[18]}$ black phosphorus, ${ }^{[19]}$ graphene, ${ }^{[20,}$ ${ }^{21]} \mathrm{MoS}_{2},{ }^{[22-25]} \mathrm{MoO}_{3},{ }^{[26]} \mathrm{h}-\mathrm{BN},{ }^{[27]}$ thin films of semiconducting oxides and sulphides, ${ }^{[28-31]}$ and organic-inorganic hybrids ${ }^{[32]}$ has led to exciting progress in bio-inspired electronics. However, to effectively mimic the biological synapses by achieving excitatory and inhibitory action potentials, these devices continue to rely on the need for simultaneously altering the polarity of the applied electric fields. This makes the development of a truly biomimetic neuromorphic system challenging, as under in vivo conditions, the polarity of the electrical signal (i.e., post-synaptic potential) is rather consistent. ${ }^{[33]}$ Parallel efforts have revealed the ability of phase change materials ${ }^{[16]}$ and light sensitive chalcogenide alloys ${ }^{[31]}$ to mimic synaptic behaviors in an all-optical stimuli set-up; however the output of these devices is a photonic synapse, instead of an electrical synapse. As the next big step towards practical realization of neuromorphic circuits that exhibit fidelity and flexibility of biological synapses, we show sole optically-stimulated synaptic devices without a need to apply polarity-altering electric field. The main hurdle so far to achieve this capability has been the lack of materials with inherent photoresponse to optically emulate both excitatory and inhibitory action potentials.

Here we report an artificial optical synapse based on few-layer black phosphorus (BP) which can imitate inhibitory and excitatory action potentials directly by optical stimulation, without the need for an additional alternating-polarity electrical stimuli. BP has recently emerged as an exciting elemental two-dimensional (2D) material with exotic electronic and optoelectronic properties that are easily tuneable. ${ }^{[34-37]}$ The broadband photoabsorption in BP, covering a wide 
spectral range from ultraviolet (UV) to infrared, ${ }^{[38-40]}$ and strong light-matter coupling ${ }^{[41]}$ render it as an ideal 2D material for optical synapses where synaptic weight can be manipulated by optical spikes of different wavelengths. We exploit oxidation-related defects in BP to achieve unique photoresponse under different $\mathrm{UV}$ wavelengths, i.e., positive (increasing) photocurrent at $280 \mathrm{~nm}$ illumination and negative (decreasing) photocurrent for a $365 \mathrm{~nm}$ excitation.

Subsequently, we exploit this unique photoresponse of BP to optically mimic excitatory and inhibitory action potentials. As such, the inherent repeatable photoconductivity of BP under UV and visible excitation wavelengths is employed to mimic a wide variety of synaptic functions, such as transition of short-term memory to long-term memory, psychological learning and forgetting, pulsed-pair facilitation, spatiotemporally correlated dynamic logic, Hebbian and associative learning for decision making. We show that this light-stimulated neuro-mimicking capability of our artificial synapse is independent of the substrate and can be extended on a flexible platform rendering it suitable for wearable and conformable electronics. Such a presentation of light triggered BP-based synapse can lead to the development of novel devices for future photonic neuromorphic circuits and synthetic sensory organs, such as artificial retina.

\section{RESULT AND DISCUSSION}

Optical synaptic devices are fabricated in a simple phototransistor configuration by mechanically exfoliating few-layer BP flakes from a bulk single crystal onto the substrates and patterning metal $(\mathrm{Cr} / \mathrm{Au})$ contacts for measuring conductivity of the devices, as schematically depicted in Figure 1a. The characterization of as-exfoliated BP flakes assessed by transmission electron microscope (TEM) and energy-dispersive X-ray spectroscopy (EDS) show absorption of oxygen on the surface (Note S1, Supporting Information) forming native phosphorus oxide $\left(\mathrm{PO}_{x}\right)$ 
layers on the as-exfoliated BP flakes which play an important role in defining photoresponse in our BP synaptic devices, as discussed later in the manuscript. To imitate different synaptic functions, optical pulses of $280 \mathrm{~nm}, 365 \mathrm{~nm}$ and $660 \mathrm{~nm}$ wavelengths are illuminated on the few-layer BP synaptic devices. Thin BP flakes (with thicknesses ranging in 5-15 nm) are identified by optical microscope and atomic force microscope (AFM). Figure $1 \mathrm{~b}$ shows an optical microscopic photograph of a representative BP synaptic device fabricated on $\mathrm{SiO}_{2} / \mathrm{Si}$ substrate with a flake of $\sim 9.2 \mathrm{~nm}$ thickness, as measured by the topological AFM scan (Figure 1c,d).

Figure 1e shows a cross-sectional TEM micrograph of a representative BP synaptic device and existence of $\sim 3 \mathrm{~nm}$ amorphous layers at top $\mathrm{Cr} / \mathrm{BP}$ and bottom $\mathrm{BP} / \mathrm{SiO}_{2}$ interfaces of the flake. To clearly identify the composition of these amorphous layers, EDS line-scan and electron energy loss spectroscopic (EELS) area maps of a region-of-interest (ROI) are acquired. The elemental signals $(\mathrm{Cr}, \mathrm{O}$ and $\mathrm{P})$ collected along the EDS line-scan (Figure 1f) across the BP synaptic device show high $\mathrm{Cr}$ and $\mathrm{P}$ intensities in $\mathrm{Cr}$ and crystalline $\mathrm{BP}$ layers, respectively. There is negligible $\mathrm{O}$ intensity in the BP layer while the interfacial regions (marked by dashed lines in Figure 1f) show signals of $\mathrm{O}$ and $\mathrm{P}$, indicating that interfacial regions are phosphorus oxide. Presence of $\mathrm{O}$ in $\mathrm{Cr}$ layer indicates partial oxidation of $\mathrm{Cr}$ to chromium oxide during sample fabrication. The EELS $\mathrm{O}-K$ edge and P- $L$ edge area maps (Figure 1g) of the ROI (marked in Figure 1e) also show relativley higher oxygen content at the interfacial regions, further confirming presence of phosphorus oxide at top and bottom interfaces of the BP synaptic device.

Stimulation of neuronal activities by different optical methods is well known in neuroscience. ${ }^{[42-44]}$ Excitatory and inhibitory photoresponse of few-layer BP synaptic devices under different excitation wavelengths is analogous to the neuronal activities triggered by light in different biological neurons. As such BP provides a platform to optically mimic synaptic functions 
of light-sensitive neurons, such as those of retinal ganglion cells (RGCs). Human brain receives sensory information through the perceptual organs for cognitive processes. Visual feed obtained from the RGCs is transferred to the frontal, temporal and occipital lobes of the brain where visual input is processed and stored, ${ }^{[45-47]}$ as schematically depicted in Figure 2a. In a biological neuronal system, neurons communicate through electrical and chemical synapses and transfer information in the form of electrical signals which are in the range of few tens of millivolts. ${ }^{[48]}$ To imitate synaptic functions of typical RGCs (which receive optical excitatory and inhibitory synaptic inputs and transfer them to the brain), we use optical stimuli with different wavelengths as synaptic spikes in our two-terminal BP synaptic devices (as schematically illustrated in Figure 1a). A small bias (50 $\mathrm{mV}$ which is on the scale of neuronal signals) is applied across the terminals to measure change in conductance of the BP channel (representing synaptic weight) under photonic excitations. Interestingly, BP inherently exhibits a strong persistent photocurrent (PPC) under UV excitations. ${ }^{[40,49,50]}$ Figure $2 \mathrm{~b}$ shows a typical transient photoresponse of the few-layer BP under UV $(280 \mathrm{~nm})$ exposure $\left(3 \mathrm{~mW} \mathrm{~cm}{ }^{-2}\right)$. The current increases upon UV exposure which can be attributed to the electron-hole pair generation after UV absorption and subsequent band-to-band excitations. This positive photocurrent imitates the excitatory post-synaptic action potential. A slow decay ( $>21 \mathrm{~s}$ ) of the photocurrent after $280 \mathrm{~nm}$ exposure is attributed to the PPC behavior of BP (Note S2 in Supporting Information) which is essential for the imitation of light-induced synaptic plasticity and memory-like behaviors.

Figure 2c schematically illustrates the psychological memory and forgetting model of a human brain, originally proposed by Atkinson and Shiffrin. ${ }^{[51]}$ According to this model, all of the information (including frequent and intermittent events) received by the perceptual organs is registered in the sensory memory. The information received with attention is converted to the 
short-term memory (STM) and is stored for a short time (from few seconds to few minutes). If frequent maintenance rehearsals are provided, it leads to learning where STM is transferred to the long-term memory (LTM) which can last from minutes to years. It should be noted that for the learning process, rehearsal stimulations should be repeated after short time intervals (on the scale of a few seconds). Longer time intervals result in STM or even memory loss i.e., forgetting. This suggests that synaptic plasticity of neurons is associated with the history of rehearsal-stimulation frequency. As such, the phenomena of synaptic plasticity and transformation of sensory information to STM and then LTM can be mimicked in our few-layer BP synaptic devices via short optical spikes with different time intervals - denoting frequency of rehearsal stimulations. At the exposure of a short optical pulse, postsynaptic current (PSC) of the BP synaptic device rapidly changes and (in dark) slowly decays back to its original level which is analogous of a biological synaptic potential. Depending on the rehearsal stimulation frequency in a given period of time, the magnitude and relaxation time of PSC vary, which is interpreted as memory in our BP synaptic devices. As shown in Figure 2d, a short optical pulse (of $280 \mathrm{~nm}$ wavelength, pulse width of $100 \mathrm{~ms}$ and power intensity of $3 \mathrm{mWcm}^{-2}$ ) acting as a stimulation event produces an excitatory PSC with change in conductance (synaptic weight) of $\sim 6.4 \%$ and relaxation time of $<20 \mathrm{~s}$, mimicking sensory memory. As the stimulation frequency increases, the synaptic weight change and decay time increase. Such as, Figure 2e shows that consecutive short optical excitatory spikes at low rehearsal stimulation frequency (of $0.2 \mathrm{~Hz}$, pulse width of $100 \mathrm{~ms}$ and power intensity of $3 \mathrm{mWcm}^{-2}$ ) over a stimulation period of $15 \mathrm{~s}$ induce STM behavior where PSC changes to $\sim 14.3 \%$ and recovers back to its initial (pre-spike current) level in $\sim 45 \mathrm{~s}$. On the other hand, optical spikes with the same intensity and pulse width but with higher rehearsal stimulation frequency (of $1 \mathrm{~Hz}$ ) over the same period of stimulation induce LTM behavior where the PSC changes to $31.5 \%$ and 
takes a comparatively longer time $(\sim 75 \mathrm{~s})$ to recover after the rehearsal stimulations are stopped (Figure 2f).

In a biological postsynaptic neuron, summation of synaptic potentials results in either excitatory postsynaptic current (EPSC) or inhibitory postsynaptic current (IPSC) which determine increase or decrease in the synaptic weight, respectively. ${ }^{[52]}$ Unlike the recently reported photonic synapses, ${ }^{[21,24]}$ where one of the two synaptic potentials is imitated by electrical signals, we utilize distinct wavelengths of the UV spectra to induce both EPSC and IPSC in our few-layer BP synaptic devices. The excitation of few-layer BP with a $280 \mathrm{~nm}$ optical stimulus induces positive photocurrent (mimicking EPSC) whereas $365 \mathrm{~nm}$ induces negative photocurrent (mimicking IPSC), as discussed in Note S3 of Supporting Information. Typically negative photoconductivity in $2 \mathrm{D}$ systems can be associated with the bolometric effect. ${ }^{[53-57]}$ However, the unusual negative photocurrent observed under longwave UV (365 nm) excitation, in the case of BP is intrinsically material-related and is associated with the surface adsorbates. ${ }^{[53,58]}$ It is well known that BP in ambient conditions readily adsorbs oxygen and forms phosphorus oxide species across the crystal surface which can act as charge trap sites. ${ }^{[58-63]}$ These extrinsic trap sites, act as scattering centers under light illumination and reduce the conductance of the device and, hence, are the origin of negative photocurrent. On the other hand, a higher energy optical stimulus such as shortwave UV $(280 \mathrm{~nm})$ results in a far higher number of photo-generated carriers which dominate over the influence of trap sites and the overall photocurrent increases. To investigate the influence of surface adsorbents on photoresponse of few-layer BP, we conducted thermal treatment of BP synaptic devices under an inert (Ar) environment (Note S4 in Supporting Information). It is observed that annealing of BP in Ar, significantly reduces these adsorbents and devices exhibit positive photoresponse under $365 \mathrm{~nm}$ excitation (Note S4 in Supporting Information). The 
evolution of oxidative species on BP (subjected to ambient and thermal treatment in Ar) is thoroughly characterized by a combination of in-situ Raman spectroscopy, X-ray photoelectron spectroscopy and energy-dispersive X-ray spectroscopy (Note S4 in Supporting Information). Furthermore, theoretical calculations also indicate that the $365 \mathrm{~nm}$ closely matches the oxidation potential of $\mathrm{BP} \cdot{ }^{[58]}$ As a result, it is expected that exposure to $365 \mathrm{~nm}$ will also result in the formation of oxidative species on the surface more readily than any other wavelength. From a practical device perspective, there are simple ways to minimize any further physical damage (i.e., degradation) by sequestering these species using an ionic liquid treatment approach as demonstrated in our previous studies. ${ }^{[59,64]}$

In neurophysiology, it is well established that stimuli duration affects the modulation of synaptic response. ${ }^{[65]}$ In this context, temporal response of both EPSC and IPSC induced in our optical synapse can be exploited to imitate spike-duration-dependent-plasticity (SDDP). The SDDP is an important feature that enables psychological processes such as learning and forgetting. The spike-duration $(t)$ dependent EPSC (Figure 3a) and IPSC (Figure 3b) shows that excitatory and inhibitory responses increase with increasing spike duration. This increasing change in PSCs (i.e., $\triangle \mathrm{EPSC}$ and $\triangle \mathrm{IPSC}$ shown in the insets of Figure $3 \mathrm{a}$ and $3 \mathrm{~b}$, respectively) can be attributed to time-dependent generation of photo-carriers in BP. Shorter (longer) optical pulses provide less (high) cumulative excitation energy which results in the generation of lesser (higher) photogenerated charge carriers and low (high) photocurrent amplitudes.

As previously mentioned, frequently administered excitatory rehearsal stimulations cause the consolidation of memory and achieve psychological learning experience. On the other hand, consecutive inhibitory synaptic potentials lead to the weakening of the synaptic connections and consequently cause loss in memory (i.e., psychological forgetting). In our BP photonic synapse, 
such temporal behaviors of memory retention are realized by repeated exposure of BP channel to optical pulses of $280 \mathrm{~nm}$ and $365 \mathrm{~nm}$ wavelengths with fixed intensity, width and rate $\left(3 \mathrm{~mW} \cdot \mathrm{cm}^{-}\right.$ ${ }^{2}, 200 \mathrm{~ms}$ and $2.5 \mathrm{~Hz}$ respectively). Figure $3 \mathrm{c}$ shows an increase in normalised EPSC as a function of the number of excitatory presynaptic optical spikes $(280 \mathrm{~nm})$ which is analogous to the learning process in a human brain. The learning behavior in our artificial photonic synapse follows a simple growth exponential function:

$$
y=y_{0}+A \cdot \exp \left(N / \tau_{1}\right)
$$

where $N$ is the number of presynaptic spikes, $A$ is initial facilitation magnitude in learning process, $y_{0}$ is resting facilitation magnitude and $\tau_{1}$ is learning time in our photonic synapse. The learning curve (Figure 3c) shows that fast learning happens at the initial stage of rehearsal stimulations and then gradually saturates with increasing number of rehearsals. This suggests that after the first few rehearsals, lesser number of stimulations is required to retain the memory. Figure $3 \mathrm{~d}$ shows decay in the normalised IPSC under inhibitory optical spikes (365 nm) and can be correlated to the Ebbinghaus' forgetting curve. ${ }^{[66]}$ The decay phenomenon of IPSC is fitted with an exponential function: ${ }^{[67,68]}$

$$
y=y_{0}+A \cdot \exp \left(-N / \tau_{2}\right)
$$

where $\tau_{2}$ is forgetting rate. The imitation of brain-like learning and forgetting processes in our BP synaptic devices by optical stimulation renders the capability of such optical synapses for artificial intelligence.

Now we explore the dynamic synaptic plasticity in our few-layer BP photonic synapses. When two presynaptic spikes separated by a period of time occur consecutively, the change in postsynaptic weight is larger for the second spike. This phenomenon is known as pair-pulse- 
facilitation $(\mathrm{PPF}){ }^{[69]}$ However, the magnitude of second change in postsynaptic weight is determined by the time interval between two consecutive spikes wherein a smaller time interval induces larger weight change. The EPSC and IPSC induced by two successive excitatory and inhibitory optical pulses (with similar pulse width of $200 \mathrm{~ms}$, time interval of $400 \mathrm{~ms}$ and power intensity of $3 \mathrm{~mW} \cdot \mathrm{cm}^{-2}$ ) are shown in Figure 3e and 3f, respectively. Herein, change in both EPSC and IPSC is higher for the second optical pulse, imitating PPF phenomenon in our few-layer BP photonic synapse. ${ }^{[69]}$ To quantify EPSC and IPSC induced PPF, the PPF index - ratio between the amplitude of second PSC $\left(A_{2}\right)$ and first PSC $\left(A_{1}\right)$ - is extracted and plotted as a function of time interval $(\Delta t)$ between consecutive pulses in Figure $3 \mathrm{~g}$ and $3 \mathrm{~h}$, respectively. The experimental PPF indexes extracted for our BP photonic synapses follow a bi-phase behavior fitted by the double exponential function given in Equation 3;

$$
y=y_{0}+A_{1} \cdot \exp \left(-\Delta t / \tau_{1}\right)+A_{2} \cdot \exp \left(-\Delta t / \tau_{2}\right)
$$

where $\Delta t$ is time interval between two consecutive optical pulses, $A_{1}$ and $A_{2}$ are facilitation magnitudes, and ${ }^{\tau_{1}}$ and ${ }^{\tau_{2}}$ are fast and slow decay times of the exponential function, respectively. In a biological synapse, PPF is caused by the residual $\mathrm{Ca}^{2+}$ ion concentration left by first spike in the presynaptic neuron which increases total $\mathrm{Ca}^{2+}$ ion level after the second spike. The residual $\mathrm{Ca}^{2+}$ ions exhibit exponential decay after the first spike which warrants lesser change in the PSC when time interval between the two spikes increases. ${ }^{[69]}$ Analogous to $\mathrm{Ca}^{2+}$ residue in biological synapses, PPF in our photonic synapse can be associated with the recombination of photogenerated carriers (i.e., relaxation time). At the end of the first optical pulse, electron-hole pairs start to recombine. If second optical pulse is exposed after a short time interval, the photogenerated electrons will not have enough time to recombine completely and charge carriers generated by the 
second pulse are augmented which consequently induces PPF in the few-layer BP. However, if the second optical spike occurs with time interval larger than relaxation time, then photocurrent has similar magnitude to the first optical spike. This suggests that paired pulses with short time interval induce higher PPF index while it saturates at $100 \%$ for paired pulses with time interval equal or higher than relaxation time. Figure 3g,h show that PPF indexes saturate for $\Delta t>70 \mathrm{~s}$ (for $280 \mathrm{~nm}$ illumination presynaptic pulses with $200 \mathrm{~ms}$ pulse-width and $3 \mathrm{mWcm}^{-2}$ power density) and $\Delta t>4 \mathrm{~s}$ (for $365 \mathrm{~nm}$ illumination pulses with $200 \mathrm{~ms}$ pulse-width and $3 \mathrm{mWcm}^{-2}$ power density), respectively.

The PPF phenomenon can be further extended to explore synaptic information processing such as temporal-frequency filtering. ${ }^{[70,71]}$ The short-term synaptic depression represents low-pass temporal filtering while short-term facilitation can interpret high-pass temporal frequency filtering. ${ }^{[70]}$ As described above, presynaptic spikes with short time interval (i.e., high-frequency stimulation) encourage postsynaptic facilitation which can realize high-pass temporal filtering. On the other hand, presynaptic spikes with longer time interval (i.e., low-frequency stimulation) induce postsynaptic depression which can realize low-pass temporal filtering. The PSC gain - ratio between $A_{10}$ and $A_{1}$ - for a train of consecutive optical pulses increases with increasing frequency indicates a high-pass temporal filtering functionality in our BP photonic synapses and control of such dynamic functionality with the stimulation frequency (Note 5 in Supporting Information).

In a multi-synaptic system, formation of new synaptic connections and strength of active synapses depend on the number and timing of spikes that two (pre- and post-) synaptic neurons trigger together. As such, spatiotemporally correlated inputs from multiple pre-synaptic neurons integrate (temporally or spatially) to trigger dynamic logic in the postsynaptic neuron. ${ }^{[72,}{ }^{73]} \mathrm{In}$ order to emulate spatiotemporal dynamic function in our optical synaptic devices employing 
pristine-like BP (exfoliated and preserved in an Ar environment to avoid surface oxidation), we utilize $660 \mathrm{~nm}$ and $280 \mathrm{~nm}$ wavelengths as pre-synapse 1 and pre-synapse2, respectively. Although due to enhanced charge separation efficiency of a hybrid electrical/optical approach ${ }^{[21,23,28]}$ (where electrical pulses are also applied on the gate terminal), we expect better synergy in imitating synaptic functions. However, an all-optical approach where dual (or multiple) optical pulses imitate synaptic functions offers the advantages of simpler operation and device architecture. Furthermore, owing to the strong light-matter interaction in BP, our synaptic devices induce high enough photoresponse (change in conductance) to imitate synaptic functions without the application of voltage pulses on the gate electrode. As shown in Figure 4b, the positive photoresponse of pristine-like BP synaptic devices, under $660 \mathrm{~nm}$ excitation, can be associated with the significantly less number of defect states, ${ }^{[60]}$ as disused in Note S6 of Supporting Information. Furthermore, previous studies have also shown a positive photocurrent under $633 \mathrm{~nm}$ and $640 \mathrm{~nm}$ excitations. ${ }^{[39,74,75]}$ Figure $4 \mathrm{~b}$ shows that an optical pulse of $660 \mathrm{~nm}$ (with pulse width of $200 \mathrm{~ms}$ and power intensity of $10 \mathrm{mWcm}^{-2}$ ) as pre-synapse1 induces a smaller change in PSC $(\triangle \mathrm{PSC} 1=\sim 2.2 \%)$ as compared to $280 \mathrm{~nm}(\triangle \mathrm{PSC} 2=\sim 13.4 \%$ with pulse width of $200 \mathrm{~ms}$ and power intensity of $3 \mathrm{mWcm}^{-2}$ ) as pre-synapse2. A relatively lower magnitude of photoresponse in few-layer BP at $660 \mathrm{~nm}$ excitation compared to UV excitation can be associated with the smaller photon energy and low absorbance of wavelengths larger than $500 \mathrm{~nm} \cdot{ }^{[39,60,76]}$

When pre-synapse 1 and pre-synapse2 are triggered sequentially with time interval (ranging from 0 to $50 \mathrm{~s}$ ), the photocarriers generated by first optical spike are added to the photocarriers generated by second optical spike which increases amplitude of the final PSC. However, this spatiotemporal summation i.e., the change in PSC ( $\triangle \mathrm{PSC}$ measured at the trailing edge of the

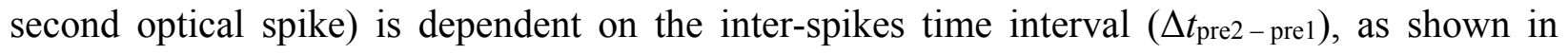


Figure $4 \mathrm{c}$. When $\Delta t_{\mathrm{pre}}$ - pre1 $=0$ i.e., Pre-synapse1 and Pre-synapse2 are triggered simultaneously, the $\triangle \mathrm{PSC}$ is maximum $(26.2 \%)$ which is higher than individual $\triangle \mathrm{PSC} 1(\sim 2.2 \%)$ and $\triangle \mathrm{PSC} 2$ $(\sim 13.4 \%)$. Furthermore, with increasing $\left|\Delta t_{\text {prez-pre1 }}\right|$ the $\Delta$ PSC decreases asymmetrically (Figure 4c). This asymmetric characteristic of $\Delta \mathrm{PSC}$ as a function of ${ }^{\left|\Delta t_{\text {prez-prel }}\right|}$ can be associated with difference in change in PSC induced by pre-synapse1 and pre-synapse2 (as shown in Figure 4b). This implies that the spatiotemporal PSC summation in our few-layer BP-based multisynaptic network exhibits super-linear amplification which is analogous to the hippocampal neurons. ${ }^{[77]}$

Utilizing the broad band absorption of few-layer BP, we optically emulate classical conditioning according to the Pavlov's dog conditioning rule ${ }^{[78]}$ as an example of associative learning in our pristine-like BP synaptic devices. Recently, John et al. ${ }^{[23]}$ implemented the classical conditioning in a $2 \mathrm{D} \mathrm{MoS}_{2}$ based transistor by coupled optical pulses as unconditioned stimuli and voltage pulses at back-gate as conditioned stimuli. Here in our work, we use solely optical pulses of $280 \mathrm{~nm}$ and $660 \mathrm{~nm}$ wavelengths as unconditioned and conditioned stimuli, respectively. The illumination of 10 consecutive pulses of $660 \mathrm{~nm}$ (with pulse width of $200 \mathrm{~ms}$, pulse interval of $100 \mathrm{~ms}$ and power intensity of $10 \mathrm{mWcm}^{-2}$ ) simulate the bell-ringing/conditioned stimulus while illumination of 10 consecutive pulses of $280 \mathrm{~nm}$ (with pulse width of $200 \mathrm{~ms}$, pulse interval of $100 \mathrm{~ms}$ and power intensity of $3 \mathrm{mWcm}^{-2}$ ) mimic the food-sight/unconditioned stimulus activating unconditioned response of salivation in the post-synapse. As depicted in Figure 4d, application of only bell-ringing/conditioned stimulus results in a slight increase $(\sim 3.4 \%)$ in PSC which is less than threshold for salivation set at $3.12 \mu \mathrm{A}$ in our experiments. While, the food-sight/unconditioned stimulus induces relatively higher change $(\sim 11 \%)$ in PSC than 
bell-ringing/conditional stimulus (i.e., $>3.12 \mu \mathrm{A}$, corresponding to salivation response). During the training phase, both bell-ringing/conditioned and food-sight/unconditioned stimuli are applied simultaneously to induce larger change in PSC. After the training stage, only bell-ringing/conditioned stimulus results in a higher amplitude of PSC than salivation threshold $(>3.12 \mu \mathrm{A})$ which imitates salivation response from the Pavlov's dog on bell-ringing. It should be noted that bell-ringing/conditioned stimulus must be applied within $70 \mathrm{~s}$ of training stimuli to induce salivation response. The increase of PSC by conditioned stimulus $(660 \mathrm{~nm})$ above salivation threshold can be associated with the augmentation of photocarriers after the training pulses.

It is well known that in-vivo neurons exhibit Hebbian synaptic learning through spike-timedependent-plasticity (STDP), ${ }^{[79]}$ which can be emulated by controlling the timing between presynaptic action potentials and postsynaptic action potentials. In STDP, the synaptic weight determined by timing difference and stimulation order of pre- and postsynaptic spikes defines asymmetric STDP. While, the synaptic weight determined only by timing difference defines symmetric STDP. H. Tian et al.$^{[19]}$ electrically emulated asymmetric STDP by using voltage pulses with opposite polarities on the gate terminal of a BP synaptic device. In our few-layer BP synaptic devices, not only asymmetric STDP is electrically imitated by using voltage pulses on the backgate terminal (see Supporting Information, Figure S7, Note S7), but also asymmetric STDP is optically emulated by illuminating $280 \mathrm{~nm}$ and $365 \mathrm{~nm}$ pulses to induce potentiation and depression, respectivley (Note S7, Figure S8). To optically emulate symmetric STDP, two separate BP synaptic devices are fabricated on the same sample and configured as shown in Figure 4e. In this configuration - sharing a common electrode - one device is referred as presynaptic neuron and the other as postsynaptic neuron. Additionally, the time difference ( $\Delta t_{\text {post- }}$ 
pre) is controlled by varying the stimulation time interval between two optical pulses (with wavelength of $280 \mathrm{~nm}$, pulse width of $200 \mathrm{~ms}$ and power intensity of $3 \mathrm{mWcm}^{-2}$ ). The synaptic weight change ( $\triangle \mathrm{PSC})$ as a function of $\Delta t_{\text {post-pre }}$ is defined as $\Delta \mathrm{PSC}=$ $\Delta \mathrm{PSC}_{\text {post }}-\Delta \mathrm{PSC}_{\text {pre }} / \Delta \mathrm{PSC}_{\text {pre }}$, plotted in Figure $4 \mathrm{f}$. The time varying optical stimulation of preand postsynaptic neurons induces a symmetric synaptic weight change regardless of the temporal order of pre- and postsynaptic spikes. Similar to the mechanism explained for Figure 4c, $\triangle \mathrm{PSC}$ is maximum for minimum $\left|\Delta t_{\text {post-pre }}\right|$ and symmetrically decreases with increasing $\left|\Delta t_{\text {post-pre }}\right|$. Although for a large scale neural network and neuromorphic computation several BP synaptic devices in a configuration compatible with peripheral CMOS circuitry such as an array of synaptic devices is essential. As such, a simple demonstration of two BP synaptic devices optically imitating a fundamental Hebbian learning (i.e., STDP) highlights the potential adoptability of these devices for future photonic neuromorphic systems.

Furthermore, we explore the optical imitation of synaptic functionality in few-layer BP synaptic devices on a flexible polyethylene naphthalate (PEN) substrate. Flexible Synaptic devices are fabricated in similar configuration as for rigid devices (shown above in Figure 1 of the main manuscript), i.e., two-terminal $\mathrm{Au} / \mathrm{Cr}$ electrodes are patterned on few-layer BP flakes $(<10 \mathrm{~nm})$, as shown in an optical microscope photographs in Figure 5a,b. To evaluate the functionality of these flexible few-layer BP synaptic devices subjected to mechanical strain, dynamic synaptic plasticity is optically imitated and quantified as PPF indexes. Figure $5 \mathrm{c}$ and $5 \mathrm{~d}$ show PPF imitated for both EPSC (by using $280 \mathrm{~nm}$ wavelength pulses) and IPSC (by using $365 \mathrm{~nm}$ wavelength pulses), respectively, at a flexure with diameter of $7 \mathrm{~mm}$. The comparison of PPF extracted for devices subject to flexure show no significant deviation from normal/flat state. Furthermore, our flexible few-layer BP synaptic devices exhibit similar PPF characteristics after being subjected to 
1000 bending cycles (Figure 5e and 5f). Other synaptic functions are also optically imitated in our flexible few-layer synaptic devices (not shown for brevity). These results demonstrate that our flexible BP devices demonstrate a robust performance by imitating synaptic functions without any significant deterioration after subjected to different mechanical strain settings. As such, the optical imitation of synaptic functions on a flexible substrate highlights the adoptability of our BP synaptic devices for wearable and conformable electronics.

The potential application of an artificial synapse in neuromorphic circuits places restriction on its energy consumption. Though several remarkable devices and materials emulating synaptic functions (electrically or optically) have been reported recently (listed in Table 1), still energy consumption is orders of magnitude higher than biological synapses ( $\sim 10 \mathrm{fJ}$ per activity). The electrical and optical energy consumed ( $E_{\text {ele }}$ and $E_{\text {opt }}$, respectivley) by our opto-elctronic few-layer BP synapses can be calculated by considering magnitude of response photocurrent $(I)$ to the time/duration of optical spike $(t)$ with illumination power $(P)$ at voltage $(V)$ and active area $(D)$ of the device, i.e., $E_{\text {ele }}=I \times t \times V^{[20,80]}$ and $E_{\text {opt }}=P \times t \times D \cdot .^{[81]}$ An optical stimulus of $280 \mathrm{~nm}$ wavelength with minimum illumination power of $0.4 \mathrm{~mW} \cdot \mathrm{cm}^{-2}$ and duration of $100 \mathrm{~ms}$ induces $>0.4 \%$ conductance change (high enough to emulate all synaptic functions) measured at applied voltage of $10 \mathrm{mV}$ across the BP synaptic devices with an average active area of $9.2 \pm 1.1 \mu \mathrm{m}^{2}$. As such, consuming $E_{\text {ele }}$ of $\sim 9.24 \times 10^{2} \mathrm{pJ}$ (with response photocurrent of $0.924 \mu \mathrm{A}$ ) and $E_{\text {opt }}$ of $\sim 3.5 \mathrm{pJ}$ per synaptic activity - higher than biological synapses. However, we project that the energy consumption of our few-layer BP synapses can be $<8 \mathrm{fJ}$ if active area of synaptic devices is scaled down to the smallest beam size of the illumination wavelength $(280 \mathrm{~nm})$, i.e., half of the wavelength of incident illumination (covering an active area of $\sim 1.96 \times 10^{-2} \mu \mathrm{m}^{2}$; Supporting Information, Note S8, Figure S9). The potential low energy consumption of our few-layer BP 
synapses highlights that these synaptic devices can be scaled up to build an artificial neural systems with capacity of human brain (consumes $\sim 20 \mathrm{~W}^{[23]}$ ) while constraining power consumption under the acceptable power budget (i.e., $\leq 20 \mathrm{~W}$ ).

Furthermore, the current device architecture of our proof-of-concept BP synapses (i.e., inplane phototransistor configuration) is prone to unintentional optical exposures during multi-wavelength synaptic operations and may lead to interpretation of inaccurate synaptic weight changes. For application of large-scale synaptic systems, optical crosstalk can be effectively mitigated by adopting photonic integrated-circuit approach where BP can be integrated in optical waveguides. For example Z. Cheng et al ${ }^{[16]}$ integrated a phase change material $\left(\mathrm{Ge}_{2} \mathrm{Sb}_{2} \mathrm{Te}_{5}\right)$ with silicon nitride waveguides in photonic synaptic devices. Also, N. Youngblood et al.$^{[82]}$ integrated BP in photonic waveguide to improve responsivity and reduce dark current in BP photodetectors. However, other engineering challenges including integration of optical systems with associated CMOS electronics would require viable solutions.

\section{CONCLUSION}

In summary, we have presented a novel light-stimulated optical synaptic device based on few-layer BP whose synaptic weight can be modified by optical stimuli without relying on electrical input signals. The positive photoresponse of few-layer BP under $280 \mathrm{~nm}$ and $660 \mathrm{~nm}$ illuminations and negative photoresponse under $365 \mathrm{~nm}$ illumination are utilized to mimic excitatory and inhibitory postsynaptic action potentials. The unprecedented optical tunability of synaptic plasticity demonstrates capability of our artificial synapses to imitate a variety of cognitive functions including learning and dynamic information processing. This demonstration of a scalable optical synaptic device based on a low dimensional material, compatible with 
conventional silicon and exotic flexible electronics, represents an effective approach to realize next generation optogenetics-inspired neuromorphic sensing and adaptive parallel processing networks.

\section{EXPERIMENTAL}

\section{Micro-fabrication of BP synaptic devices}

Layered BP flakes were obtained via poly-dimethyl-siloxane (PDMS) assisted mechanical exfoliation of a commercial bulk BP crystal (Smart Elements). The rigid substrates $\left(\mathrm{SiO}_{2}(300 \mathrm{~nm}) / p\right.$-doped $\left.\mathrm{Si}\right)$ and flexible $125 \mu \mathrm{m}$ polyethylene naphthalate (PEN) foils were thoroughly cleaned with acetone and isopropanol followed by blow dry in $\mathrm{N}_{2}$ and then dehydration at $150{ }^{\circ} \mathrm{C}$ for $5 \mathrm{~min}$, in case of rigid substrates. Furthermore, substrates were treated with low-power oxygen plasma $(30 \mathrm{~W})$ for $5 \mathrm{~min}$ to remove organic residue. Then exfoliated few-layer BP flakes were transferred onto the clean substrates. The BP flakes with thicknesses in the range of 5-15 nm were identified by an optical microscope and AFM. Standard photolithography/lift-off were used to pattern electrodes on thin BP flakes with different channel areas in the range of $9.1 \mu \mathrm{m}^{2}$ and $19.5 \mu \mathrm{m}^{2}$. Metal $\mathrm{Cr}(5 \mathrm{~nm}) / \mathrm{Au}(90 \mathrm{~nm})$ and $\mathrm{Ti}(5 \mathrm{~nm}) / \mathrm{Au}$ $(90 \mathrm{~nm})$ electrodes were then deposited on the developed patterns using electron beam evaporation at pressure $<5 \times 10^{-7}$ Torr. Finally, lift-off in acetone was carried out to reveal the required metallic contact pads for micro-probes and electrical measurement.

\section{Transmission electron microscopy}

The transmission electron microscopy (TEM), energy-dispersive X-ray spectroscopy (EDS) and electron energy loss spectroscopic (EELS) analyses were performed by using a JEOL 
2100F scanning transmission electron microscope (STEM) with attached Tridium Gatan image filter (aperture of $5 \mathrm{~mm}$ ). For the cross-sectional analyses, thin TEM lamellae were prepared by focused ion beam (FIB) from BP synaptic devices by using a FEI Scios DualBeam FIBSEM system. Cross-sectional STEM micrographs and EELS spectra were collected using a $<1.5 \mathrm{~nm}$ beam spot. A power law fit was adopted for P- $L$ and O- $K$ pre-edge background correction while the influence of nearby peaks and plural scattering were reduced by narrow signal windows. Elemental EDS maps were collected with a Bruker Quantax EDX system.

\section{AFM and Raman characterization}

AFM topographic scans of the exfoliated BP flakes were conducted on a Dimension-Icon AFM in ScanAsyst mode. Raman spectra were obtained by using a Horiba LabRAM Evolution micro-Raman system equipped with $9 \mathrm{~mW}, 532 \mathrm{~nm}$ laser $(0.5 \mu \mathrm{m}$ lateral resolution, $0.25 \mathrm{~s}$ exposure), and a $50 \times$ objective.

\section{X-ray photoelectron spectroscopy}

X-ray photoelectron spectroscopy (XPS) analysis was conducted by using a Thermo Scientific K-Alpha instrument utilizing an aluminum $K \alpha$ radiation source $(1486.6 \mathrm{eV})$. The XPS spectra were collected from BP flakes exfoliated on the $\mathrm{SiO}_{2} / \mathrm{Si}$ substrates. The adventitious carbon binding energy (C $1 s)$ of $285 \mathrm{eV}$ was used as reference for peak fitting of principal elements. All spectra were resolved by using the standard Gaussian-Lorentzian function followed by the Shirley background correction.

\section{Electrical and photoelectronic measurements}

Electrical measurements were conducted using Keithley 4200-SCS semiconductor parameter analyser and Keysight 2912A sourcemeter. For optoelectronic measurements, 
commercial monochromatic light-emitting diodes with illumination wavelengths of $280 \mathrm{~nm}$, $365 \mathrm{~nm}$ and $660 \mathrm{~nm}$ (from Thorlabs, Inc.) were used as excitation sources. The illumination power was calibrated by a commercial UV-enhanced silicon photodetector (Newport Corporation). The excitation frequency and pulse width of the light sources were controlled by Keysight U2961A function generator and programmable Arduino-Uno microcontroller board. The channel area dependent conductance of BP synaptic devices is measured under $280 \mathrm{~nm}$ and $365 \mathrm{~nm}$ illuminations revealing inverse relation to the channel area (Note S9 in Supporting Information). For reliable operation of optical few-layer BP synaptic devices, we employed a recently reported chemical sequestration technique based on 1-butyl-3-methylimidazolium tetrafluoroborate $[\mathrm{BMIM}]\left[\mathrm{BF}_{4}\right]$ ionic liquid to prevent the photo-degradation of few-layer $\mathrm{BP},{ }^{[64]}$ Note S10 of Supporting Information.

\section{Supporting Information}

Supporting Information is available from the Wiley Online Library or from the author.

\section{Acknowledgements}

This work was performed in part at the Micro Nano Research Facility (MNRF) at RMIT University in the Victorian Node of the Australian National Fabrication Facility (ANFF). The authors acknowledge the facilities and technical support of the staff at MNRF and the RMIT Microscopy and Microanalysis Research Facility (RMMF).

Funding: The authors acknowledge support from the Australian Research Council (ARC) for personnel and project support (DP130100062), fellowship (DE160100023, FT140101285), and infrastructure (LE0882246 and LE0989615) support.

Author contributions: T.A., S.W. conceptualized this work with inputs from S.S. and M.B. T.A., S.K. undertook device fabrication and characterization presented in this manuscript with T.A. and S.W. conducting the data analysis. E.L.H.M. performed the TEM and EDX analysis. T.A., R.R. and V.B. performed characterizations of chemical protection and XPS of black phosphorus. T.A., S.W. prepared the manuscript with inputs from all authors. 
Competing interests: The authors declare that they have no competing interests.

Data and materials availability: All data needed to evaluate the conclusions in the paper are present in the paper and/or Supplementary Materials. Additional data related to this paper may be requested from the authors.

\section{REFERENCES}

[1] M. Prezioso, F. Merrikh-Bayat, B. D. Hoskins, G. C. Adam, K. K. Likharev, D. B. Strukov, Nature 2015, 521, 61.

[2] P. A. Merolla, J. V. Arthur, R. Alvarez-Icaza, A. S. Cassidy, J. Sawada, F. Akopyan, B. L. Jackson, N. Imam, C. Guo, Y. Nakamura, B. Brezzo, I. Vo, S. K. Esser, R. Appuswamy, B. Taba, A. Amir, M. D. Flickner, W. P. Risk, R. Manohar, D. S. Modha, Science 2014, 345, 668.

[3] H. Markram, Nat. Rev. Neurosci. 2006, 7.

[4] M. Chu, B. Kim, S. Park, H. Hwang, M. Jeon, B. H. Lee, B. G. Lee, IEEE Trans. Ind. Electron. 2015, 62, 2410.

[5] J. Shi, S. D. Ha, Y. Zhou, F. Schoofs, S. Ramanathan, Nat. Commun. 2013, 4, 2676.

[6] Ö. Türel, J. H. Lee, X. Ma, K. K. Likharev, Int. J. Circ. Theor. App. 2004, 32, 277.

[7] S. Kim, B. Choi, M. Lim, J. Yoon, J. Lee, H.-D. Kim, S.-J. Choi, ACS Nano 2017, 11, 2814.

[8] P. M. Sheridan, F. Cai, C. Du, W. Ma, Z. Zhang, W. D. Lu, Nat. Nanotechnol. 2017, 12, 784.

[9] J. J. Yang, D. B. Strukov, D. R. Stewart, Nat Nanotechnol 2013, 8, 13.

[10] K. H. Kim, S. Gaba, D. Wheeler, J. M. Cruz-Albrecht, T. Hussain, N. Srinivasa, W. Lu, Nano Lett. 2012, 12, 389.

[11] J. A. Hutchby, G. I. Bourianoff, V. V. Zhirnov, J. E. Brewer, IEEE Circuits Devices Mag. 2002, 18, 28.

[12] T. Uwano, H. Nishijo, T. Ono, R. Tamura, Neurosci. 1995, 68, 339.

[13] Y. Aso, D. Hattori, Y. Yu, R. M. Johnston, N. A. Iyer, T.-T. B. Ngo, H. Dionne, L. F. Abbott, R. Axel, H. Tanimoto, G. M. Rubin, eLife 2014, 3, e04577.

[14] A. Vanarse, A. Osseiran, A. Rassau, Front. Neurosci. 2016, 10, 115. 
[15] D. A. B. Miller, Proc. IEEE 2009, 97, 1166.

[16] Z. Cheng, C. Ríos, W. H. P. Pernice, C. D. Wright, H. Bhaskaran, Sci. Adv. 2017, 3.

[17] A. N. Tait, M. A. Nahmias, Y. Tian, B. J. Shastri, P. R. Prucnal, in Nanophotonic information physics, Springer, Berlin 2014.

[18] G. Agnus, W. Zhao, V. Derycke, A. Filoramo, Y. Lhuillier, S. Lenfant, D. Vuillaume, C. Gamrat, J. P. Bourgoin, Adv. Mater. 2010, 22, 702.

[19] H. Tian, Q. Guo, Y. Xie, H. Zhao, C. Li, J. J. Cha, F. Xia, H. Wang, Adv. Mater. 2016, 28, 4991.

[20] M. T. Sharbati, Y. Du, J. Torres, N. D. Ardolino, M. Yun, F. Xiong, Adv. Mater. 2018, e1802353.

[21] S. Qin, F. Wang, Y. Liu, Q. Wan, X. Wang, Y. Xu, Y. Shi, X. Wang, R. Zhang, 2D Mater. 2017, 4, 035022.

[22] V. K. Sangwan, H.-S. Lee, H. Bergeron, I. Balla, M. E. Beck, K.-S. Chen, M. C. Hersam, Nature 2018, 554, 500.

[23] R. A. John, F. Liu, N. A. Chien, M. R. Kulkarni, C. Zhu, Q. Fu, A. Basu, Z. Liu, N. Mathews, Adv. Mater. 2018, 30, e1800220.

[24] H. K. He, R. Yang, W. Zhou, H. M. Huang, J. Xiong, L. Gan, T. Y. Zhai, X. Guo, Small $2018,1$.

[25] A. J. Arnold, A. Razavieh, J. R. Nasr, D. S. Schulman, C. M. Eichfeld, S. Das, ACS Nano $2017,11,3110$.

[26] C. S. Yang, D. S. Shang, N. Liu, G. Shi, X. Shen, R. C. Yu, Y. Q. Li, Y. Sun, Adv. Mater. 2017, 29.

[27] Y. Shi, X. Liang, B. Yuan, V. Chen, H. Li, F. Hui, Z. Yu, F. Yuan, E. Pop, H. S. P. Wong, M. Lanza, Nat. Electronics 2018, 1, 458.

[28] H. K. Li, T. P. Chen, P. Liu, S. G. Hu, Y. Liu, Q. Zhang, P. S. Lee, J. Appl. Phys. 2016, $119,244505$.

[29] M. Lee, W. Lee, S. Choi, J. W. Jo, J. Kim, S. K. Park, Y. H. Kim, Adv. Mater. 2017, 29.

[30] S. Chen, Z. Lou, D. Chen, G. Shen, Adv. Mater. 2018, 30, 1705400.

[31] B. Gholipour, P. Bastock, C. Craig, K. Khan, D. Hewak, C. Soci, Adv. Opt. Mater. 2015, 3, 635.

[32] X. Zhu, W. D. Lu, ACS Nano 2018, 12, 1242. 
[33] D. Purves, Neuroscience, Oxford University Press, 2012.

[34] Y. Saito, Y. Iwasa, ACS Nano 2015, 9, 3192.

[35] F. Xia, H. Wang, Y. Jia, Nat. Commun. 2014, 5, 4458.

[36] H. Liu, A. T. Neal, Z. Zhu, Z. Luo, X. Xu, D. Tománek, P. D. Ye, ACS Nano 2014, 8, 4033.

[37] R. Roldán, A. Castellanos-Gomez, Nat. Photon. 2017, 11, 407.

[38] X. Chen, X. Lu, B. Deng, O. Sinai, Y. Shao, C. Li, S. Yuan, V. Tran, K. Watanabe, T. Taniguchi, D. Naveh, L. Yang, F. Xia, Nat. Commun. 2017, 8, 1672.

[39] M. Huang, M. Wang, C. Chen, Z. Ma, X. Li, J. Han, Y. Wu, Adv. Mater. 2016, $28,3481$.

[40] J. Wu, G. K. W. Koon, D. Xiang, C. Han, C. T. Toh, E. S. Kulkarni, I. Verzhbitskiy, A. Carvalho, A. S. Rodin, S. P. Koenig, G. Eda, W. Chen, A. H. C. Neto, B. Özyilmaz, ACS Nano 2015, 9, 8070.

[41] J. Lu, J. Yang, A. Carvalho, H. Liu, Y. Lu, C. H. Sow, Acc. Chem. Res. 2016, 49, 1806.

[42] J.-J. Pang, F. Gao, S. M. Wu, J. Neurosci. 2003, 23, 6063.

[43] E. S. Boyden, F. Zhang, E. Bamberg, G. Nagel, K. Deisseroth, Nat. Neurosci. 2005, 8, 1263.

[44] A. C. Thompson, P. R. Stoddart, E. D. Jansen, Curr. Mol. Imaging 2014, 3, 162.

[45] J. S. Simons, H. J. Spiers, Nat. Rev. Neurosci. 2003, 4, 637.

[46] J. Fell, P. Klaver, K. Lehnertz, T. Grunwald, C. Schaller, C. E. Elger, G. Fernández, Nat. Neurosci. 2001, 4, 1259.

[47] P. E. Roland, B. Gulyás, Cereb. Cortex 1995, 5, 79.

[48] A. L. Hodgkin, A. F. Huxley, Bull. Math. Biol. 1990, 52, 25.

[49] M. B. Erande, M. S. Pawar, D. J. Late, ACS Appl. Mater. Interfaces 2016, 8, 11548.

[50] S. Kuriakose, T. Ahmed, S. Balendhran, G. E. Collis, V. Bansal, I. Aharonovich, S. Sriram, M. Bhaskaran, S. Walia, Appl. Mater. Today 2018, 12, 244.

[51] R. C. Atkinson, R. M. Shiffrin, in Psychology of learning and motivation, Vol. 2, Elsevier, $1968,89$.

[52] J. C. Eccles, The Physiology of synapses, Academic Press, 2013. 
[53] C. Biswas, F. Gunes, D. L. Duong, S. C. Lim, M. S. Jeong, D. Pribat, Y. H. Lee, Nano Lett. 2011, 11, 4682.

[54] J. Miao, B. Song, Q. Li, L. Cai, S. Zhang, W. Hu, L. Dong, C. Wang, ACS Nano 2017, 11, 6048 .

[55] L. Huang, W. C. Tan, L. Wang, B. Dong, C. Lee, K. W. Ang, ACS Appl. Mater. Interfaces $2017,9,36130$.

[56] S. Liu, N. Huo, S. Gan, Y. Li, Z. Wei, B. Huang, J. Liu, J. Li, H. Chen, J. Mater. Chem. C 2015, 3, 10974.

[57] J. Y. Wu, Y. T. Chun, S. Li, T. Zhang, J. Wang, P. K. Shrestha, D. Chu, Adv. Mater. 2018, 30.

[58] K. L. Utt, P. Rivero, M. Mehboudi, E. O. Harriss, M. F. Borunda, A. A. Pacheco SanJuan, S. Barraza-Lopez, ACS Cent. Sci. 2015, 1, 320.

[59] S. Kuriakose, T. Ahmed, S. Balendhran, V. Bansal, S. Sriram, M. Bhaskaran, S. Walia, 2D Mater. 2018, 5, 032001.

[60] T. Ahmed, S. Balendhran, M. N. Karim, E. L. H. Mayes, M. R. Field, R. Ramanathan, M. Singh, V. Bansal, S. Sriram, M. Bhaskaran, S. Walia, npj 2D Mater. Appl. 2017, 1.

[61] S. Walia, Y. Sabri, T. Ahmed, M. R. Field, R. Ramanathan, A. Arash, S. K. Bhargava, S. Sriram, M. Bhaskaran, V. Bansal, S. Balendhran, 2D Mater. 2016, 4, 015025.

[62] A. Favron, E. Gaufres, F. Fossard, A. L. Phaneuf-L'Heureux, N. Y. Tang, P. L. Levesque, A. Loiseau, R. Leonelli, S. Francoeur, R. Martel, Nat. Mater. 2015, 14, 826.

[63] J. D. Wood, S. A. Wells, D. Jariwala, K.-S. Chen, E. Cho, V. K. Sangwan, X. Liu, L. J. Lauhon, T. J. Marks, M. C. Hersam, Nano Lett. 2014, 14, 6964.

[64] S. Walia, S. Balendhran, T. Ahmed, M. Singh, C. El-Badawi, M. D. Brennan, P. Weerathunge, M. N. Karim, F. Rahman, A. Rassell, J. Duckworth, R. Ramanathan, G. E. Collis, C. J. Lobo, M. Toth, J. C. Kotsakidis, B. Weber, M. Fuhrer, J. M. Dominguez-Vera, M. J. S. Spencer, I. Aharonovich, S. Sriram, M. Bhaskaran, V. Bansal, Adv. Mater. 2017, 29.

[65] M. Im, P. Werginz, S. I. Fried, J. Neural. Eng. 2018, 15, 036010.

[66] T. Ohno, T. Hasegawa, T. Tsuruoka, K. Terabe, J. K. Gimzewski, M. Aono, Nat. Mater. 2011, 10, 591.

[67] Z. Q. Wang, H. Y. Xu, X. H. Li, H. Yu, Y. C. Liu, X. J. Zhu, Adv. Funct. Mater. 2012, 22, 2759 . 
[68] R. Yang, K. Terabe, Y. Yao, T. Tsuruoka, T. Hasegawa, J. K. Gimzewski, M. Aono, Nanotechnology 2013, 24, 384003.

[69] D. Debanne, N. C. Guérineau, B. H. Gähwiler, S. M. Thompson, J. Physiol. 1996, 491, 163.

[70] E. S. Fortune, G. J. Rose, Trends Neurosci. 2001, 24, 381.

[71] L. F. Abbott, W. G. Regehr, Nature 2004, 431, 796.

[72] J. C. Magee, Nat. Rev. Neurosci. 2000, 1, 181.

[73] N. Spruston, W. L. Kath, Nat. Neurosci. 2004, 7, 567.

[74] M. Buscema, D. J. Groenendijk, S. I. Blanter, G. A. Steele, H. S. van der Zant, A. Castellanos-Gomez, Nano Lett. 2014, 14, 3347.

[75] F. Liu, C. Zhu, L. You, S. J. Liang, S. Zheng, J. Zhou, Q. Fu, Y. He, Q. Zeng, H. J. Fan, L. K. Ang, J. Wang, Z. Liu, Adv. Mater. 2016, 28, 7768.

[76] Z. Guo, H. Zhang, S. Lu, Z. Wang, S. Tang, J. Shao, Z. Sun, H. Xie, H. Wang, X.-F. Yu, P. K. Chu, Adv. Funct. Mater. 2015, 25, 6996.

[77] M. Yoneyama, Y. Fukushima, M. Tsukada, T. Aihara, Cogn. Neurodyn. 2011, 5, 333.

[78] M. S. Fanselow, A. M. Poulos, Annu. Rev. of Psychol. 2004, 56, 207.

[79] S. Sen, K. D. Miller, L. F. Abbott, Nat. Neurosci. 2000, 3, 919.

[80] Y. Wang, Z. Lv, J. Chen, Z. Wang, Y. Zhou, L. Zhou, X. Chen, S. T. Han, Adv. Mater. 2018, e1802883.

[81] H. Tan, Z. Ni, W. Peng, S. Du, X. Liu, S. Zhao, W. Li, Z. Ye, M. Xu, Y. Xu, X. Pi, D. Yang, Nano Energy 2018, 52, 422.

[82] N. Youngblood, C. Chen, S. J. Koester, M. Li, Nat. Photon. 2015.

[83] J. Zhu, Y. Yang, R. Jia, Z. Liang, W. Zhu, Z. U. Rehman, L. Bao, X. Zhang, Y. Cai, L. Song, R. Huang, Adv Mater 2018, 30, e1800195.

[84] H. Tan, Z. Ni, W. Peng, S. Du, X. Liu, S. Zhao, W. Li, Z. Ye, M. Xu, Y. Xu, X. Pi, D. Yang, Nano Energy 2018. 


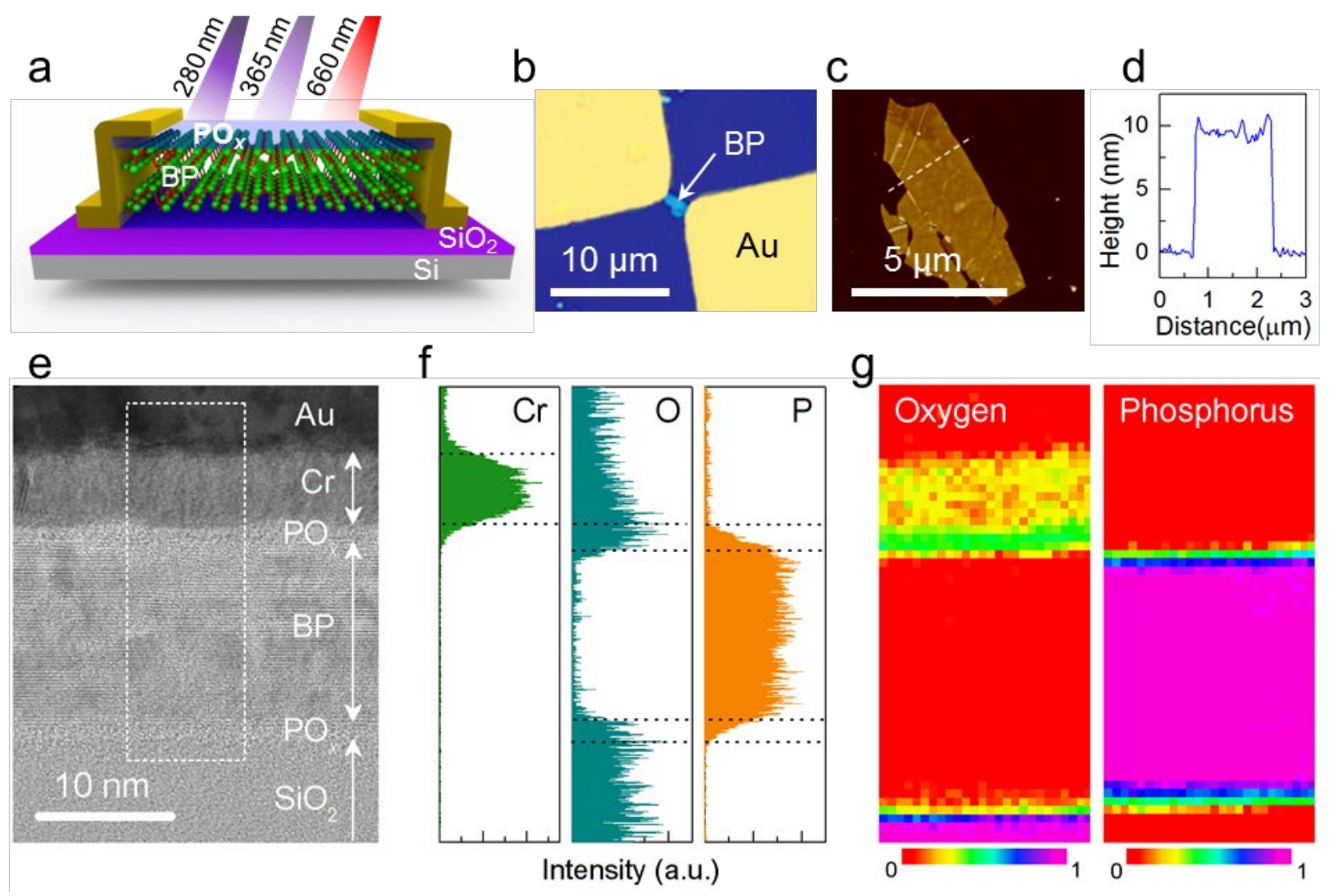

Figure 1. BP synaptic devices. (a) A schematic illustration of BP synaptic devices fabricated on a $\mathrm{SiO}_{2} / \mathrm{Si}$ substrate. Monochromatic optical pulses with $280 \mathrm{~nm}, 365 \mathrm{~nm}$ and $660 \mathrm{~nm}$ wavelengths are used as synaptic stimuli to emulate different neuronal functions. (b) An optical microscope photograph of a representative BP synaptic device. (c) Atomic force microscope (AFM) micrograph of the BP flake selected for BP synaptic device shown in (b). (d) Thickness profile of the BP flake in (c) measured along dashed line highlighted in (c). (e) Cross-sectional transmission electron microscope (TEM) micrograph of a representative BP synaptic device. (f) Elemental energy-dispersive X-ray spectroscopy (EDS) profiles for $\mathrm{Cr}, \mathrm{O}$ and $\mathrm{P}$ along a line-scan across the BP synaptic device. (g) Electron energy loss spectroscopy (EELS) area maps generated for the region of interest highlighted in (e) by taking $\mathrm{O}-K$ edge and $\mathrm{P}-L$ edge intensities of the collected spectra (at each pixel) after pre-edge background subtraction. 

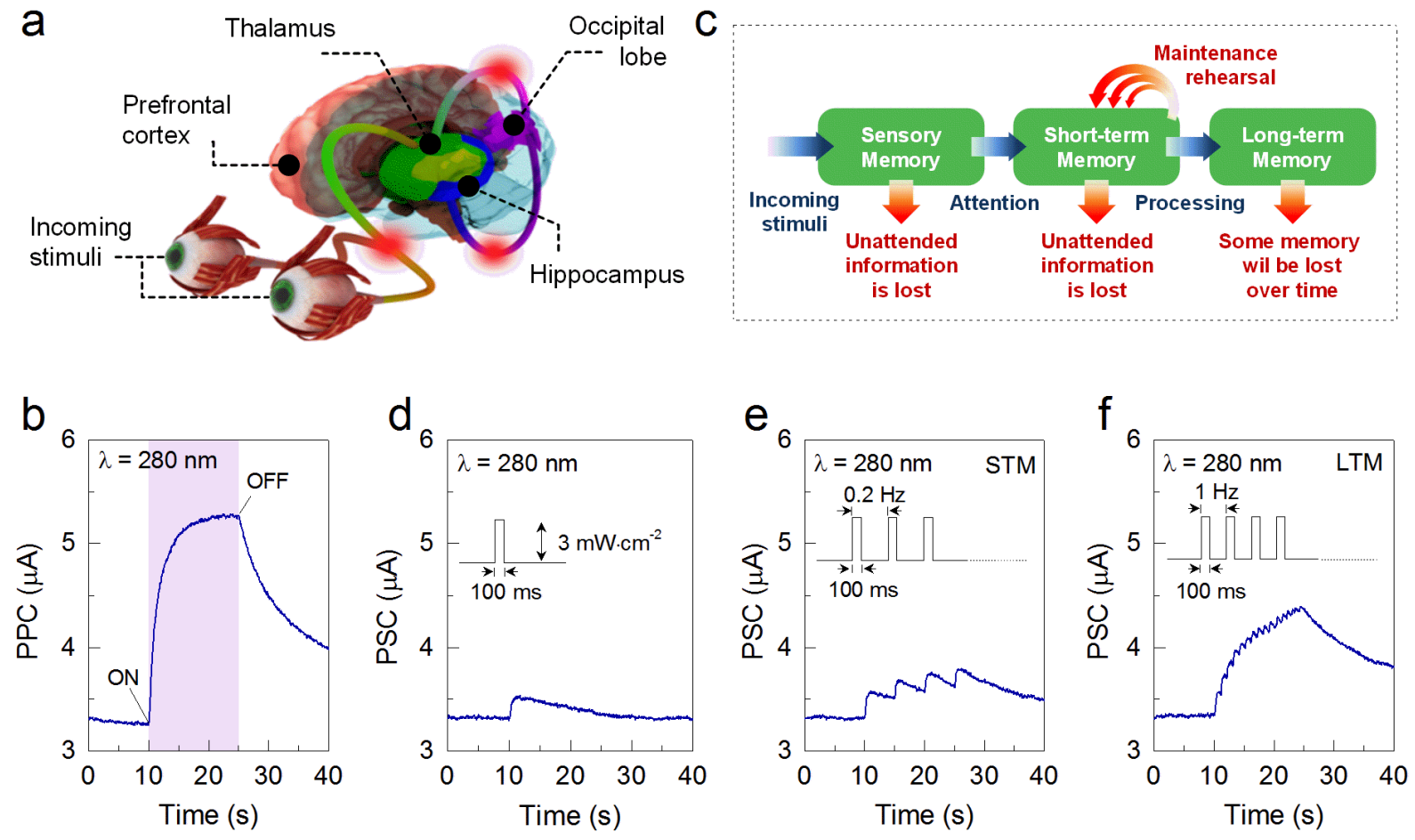

Figure 2. BP based synaptic devices and optically imitation of psychological functions. (a)

Schematic illustration of human visual system and transfer of received information from eyes to different parts of the brain. (b) Typical transient photoresponse of the BP synaptic devices under $280 \mathrm{~nm}$ excitation wavelength at intensity of $3 \mathrm{~mW} \cdot \mathrm{cm}^{-2}$. (c) A memory model illustrating shortterm and long-term memory phenomena. Optical imitation of (d) sensory memory, (e) short-term memory - STM and (f) long-term memory - LTM by excitation pulses of $280 \mathrm{~nm}$ wavelength with $100 \mathrm{~ms}$ pulse width, power intensity of $3 \mathrm{~mW} \cdot \mathrm{cm}^{-2}$ and rate of $0.2 \mathrm{~Hz}(\mathrm{STM})$ and $1 \mathrm{~Hz}$ (LTM). Insets of (d)-(f): Schematic of excitation pulses with respective pulse-parameters. 

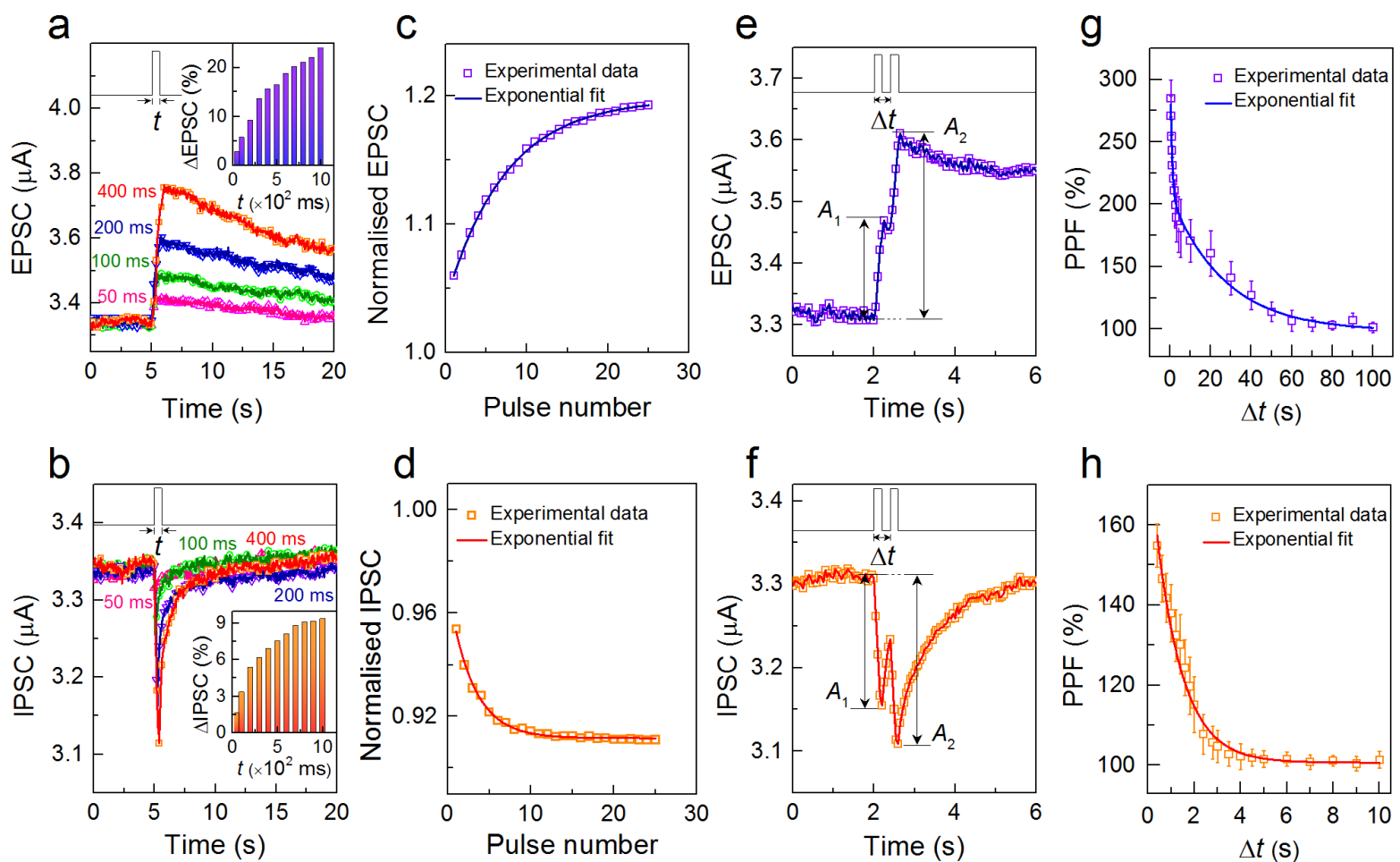

Figure 3. Optical stimulation of synaptic functions in BP synaptic devices. Transient PSC of BP synaptic devices in response to optical spike-duration $(t)$ of (a) $280 \mathrm{~nm}$ optical stimulus - emulating EPSC and (b) $365 \mathrm{~nm}$ optical stimulus - emulating IPSC. Insets of (a) and (b) show spike-duration-dependent change in EPSC and IPSC, respectively. (c) Normalised EPSC and (d) normalised IPSC as a function of number of presynaptic optical spikes with pulse width of $200 \mathrm{~ms}$, power density of $3 \mathrm{~mW} \mathrm{~cm}{ }^{-2}$ and rate of $2.5 \mathrm{~Hz}$. The change in (e) EPSC and (f) IPSC in response to the pair of presynaptic optical pulses with time interval $(\Delta t)$ of $400 \mathrm{~ms}$ and pulse width of $200 \mathrm{~ms}$. $A_{1}$ and $A_{2}$ represent the change in PSC at first and second spike, respectively. Pair pulse facilitation (PPF) index as a function of time interval between pairs of ( $\mathrm{g}$ ) excitatory and (h) inhibitory pulses. Error bars represent standard deviation in measurements for at least 5 different BP synaptic devices. 

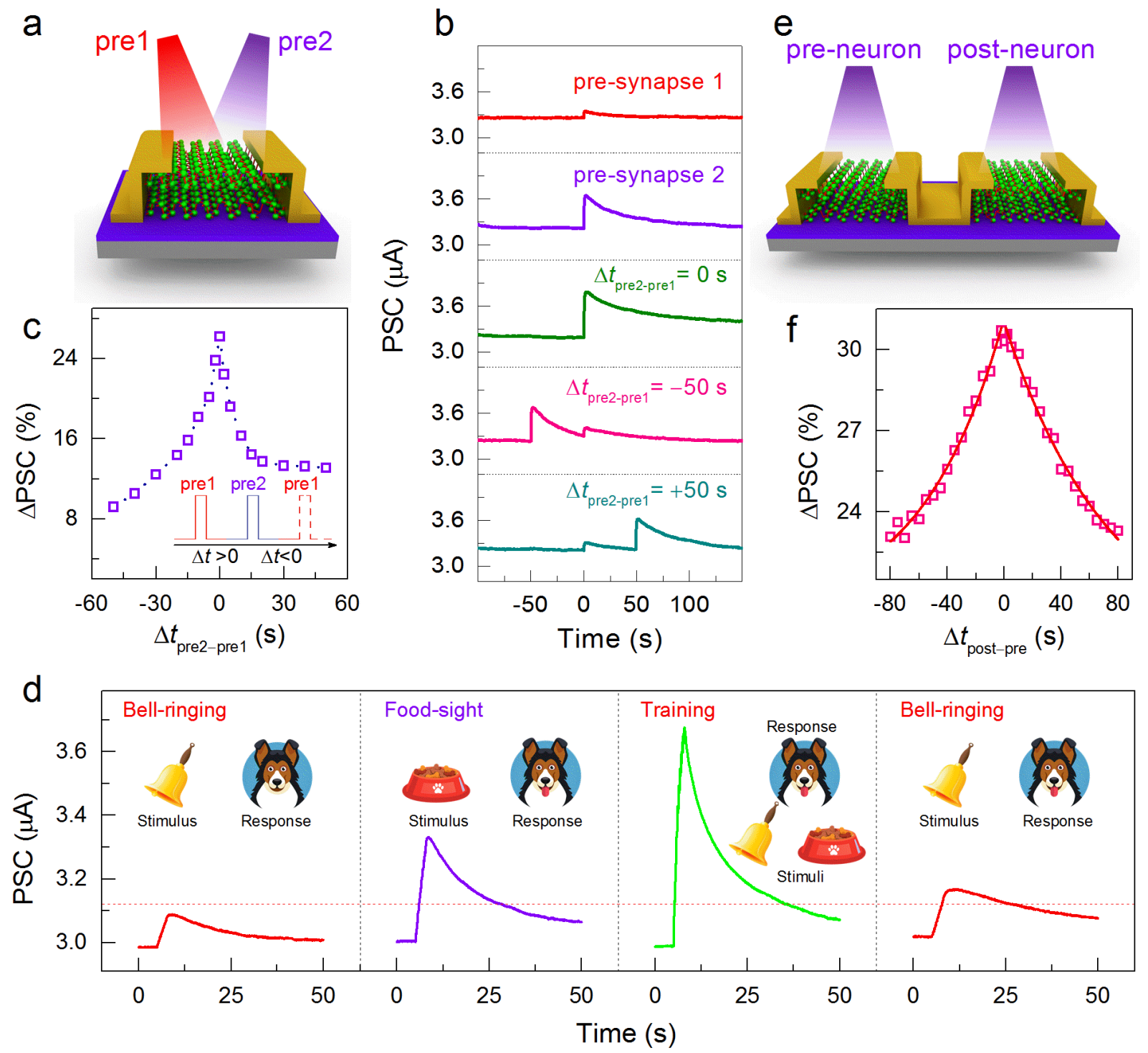

Figure 4. Optically-stimulated synaptic learning in few-layer BP multi-neural network. (a)

Schematic illustration of multi-neural system employing $660 \mathrm{~nm}$ and $280 \mathrm{~nm}$ excitation wavelengths as pre-synapse1 (pre1) and pre-synapse2 (pre2), respectively. (b) Transient PSC triggered by single or a pair of spatiotemporally correlated presynaptic optical pulses. (c) Change in PSC as a function of time interval between optical stimulations at pre-synapse 1 and pre-syapse2.(d) Imitation of classical Pavlov's learning by using optical pulses of $660 \mathrm{~nm}$ wavelength (pulse width of $200 \mathrm{~ms}$, pulse interval of $100 \mathrm{~ms}$ and power intensity of $10 \mathrm{~mW} \cdot \mathrm{cm}^{-}$ ${ }^{2}$ ) as unconditioned stimuli and optical pulses of $280 \mathrm{~nm}$ (pulse width of $200 \mathrm{~ms}$, pulse interval of $100 \mathrm{~ms}$ and power intensity of $3 \mathrm{mWcm}^{-2}$ ) as conditioned stimuli. Red dash-line represents threshold for salivation and is set at $3.12 \mu \mathrm{A}$ in our experiments. (e) A schematic illustration of 
two BP synaptic devices configured to optically imitate spike-time-dependent-plasticity (STDP) by using optical pulses of $280 \mathrm{~nm}$. (f) Change in synaptic weight ( $\triangle \mathrm{PSC}$ ) as a function of time difference between post- and presynaptic pulses ( $\left.\Delta t_{\text {post-pre }}\right)$.
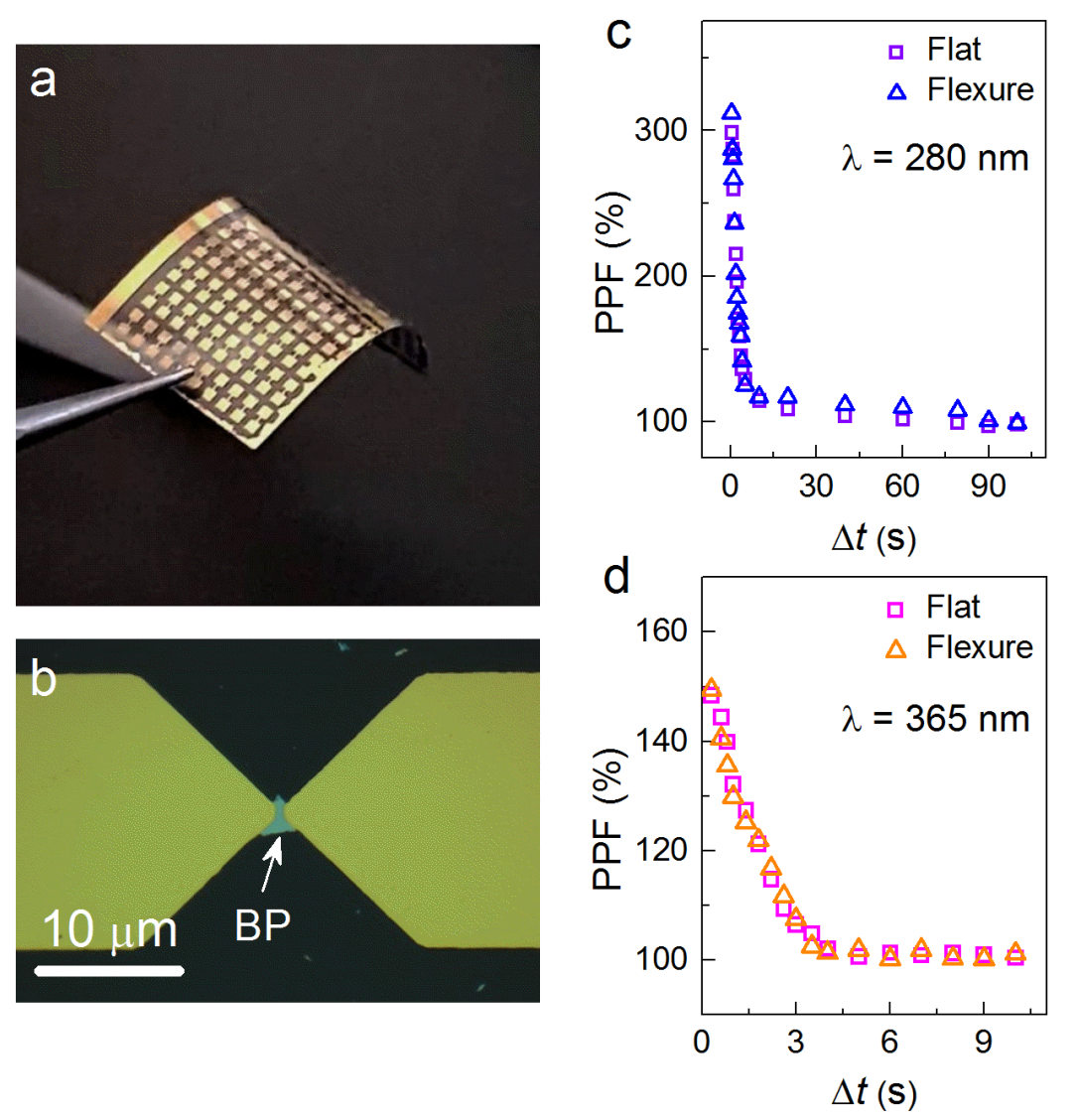
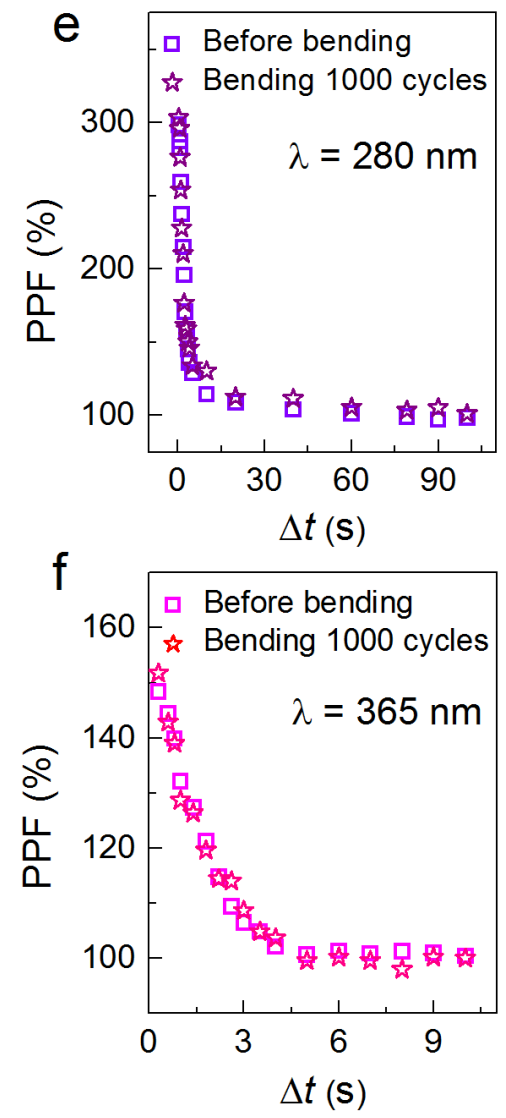

Figure 5. Flexible few-layer BP synaptic devices. (a) Photograph of flexible few-layer BP synaptic devices on a polyethylene naphthalate (PEN) substrate, showing flexibility of the substrate. (b) Optical microscope photograph of a representative two-terminal synaptic device on PEN. Imitation of PPF in flexible BP synaptic devices at flat and flexure (with diameter of $7 \mathrm{~mm}$ ) by using optical pulses of (c) $280 \mathrm{~nm}$ and (d) $365 \mathrm{~nm}$ wavelength. PPF indexes of flexible BP synaptic devices before and after subjected to 1000 bending cycles by using optical pulses of (c) $280 \mathrm{~nm}$ and (d) $365 \mathrm{~nm}$ wavelength. 
Table 1. Comparison of key features of our few-layer BP synaptic devices with reported synaptic devices. Abbreviations - PEN: Polyethylene napthalate, NG: Not given

\begin{tabular}{|c|c|c|c|c|c|c|c|c|c|c|c|}
\hline \multirow{2}{*}{\multicolumn{2}{|c|}{ Material }} & \multicolumn{2}{|c|}{ Presynaptic stimuli } & \multicolumn{4}{|c|}{ Neural functions } & \multicolumn{3}{|c|}{ Performance } & \multirow[b]{2}{*}{ Ref. } \\
\hline & & $\begin{array}{c}\text { Optical } \\
\text { (wavelength) }\end{array}$ & Electrical & $\begin{array}{l}\text { PPF index } \\
\text { (max.) }\end{array}$ & STDP & $\begin{array}{c}\text { Neural } \\
\text { logic/filtering }\end{array}$ & $\begin{array}{l}\text { Associative } \\
\text { learning }\end{array}$ & $\begin{array}{l}\text { Presynaptic } \\
\text { spike time } \\
(\mathrm{ms})\end{array}$ & $\begin{array}{c}\text { Power } \\
\text { consumption (pJ) }\end{array}$ & Substrate & \\
\hline \multirow{9}{*}{$\begin{array}{c}\text { Low } \\
\text { dimensional }\end{array}$} & \multirow[t]{2}{*}{$\begin{array}{c}\text { Black } \\
\text { Phosphorus }\end{array}$} & $\begin{array}{c}\text { Potentiation }(280, \\
660 \mathrm{~nm}) \\
\& \\
\text { Habituation } \\
(365 \mathrm{~nm}) \\
\end{array}$ & $\begin{array}{c}\text { Potentiation } \\
\& \\
\text { Habituation }\end{array}$ & $\begin{array}{l}\sim 285 \%(280 \mathrm{~nm}) \\
\sim 155 \%(365 \mathrm{~nm})\end{array}$ & Yes & Yes & Yes & $10^{2}$ & $\begin{array}{l}\sim 3.5 \text { (optical) } \\
\sim 9.24 \times 10^{2} \\
(\text { electrical }) \\
\end{array}$ & $\begin{array}{l}\mathrm{SiO}_{2} / \mathrm{Si} \\
\& \\
\mathrm{PEN}\end{array}$ & $\begin{array}{l}\text { This } \\
\text { Work }\end{array}$ \\
\hline & & -- & $\begin{array}{c}\text { Potentiation } \\
\& \\
\text { Habituation } \\
\end{array}$ & No & Yes & No & No & 10 & NG & $\mathrm{SiO}_{2} / \mathrm{Si}$ & {$[19]$} \\
\hline & \multirow[t]{2}{*}{$\mathrm{MoS}_{2}$} & $\begin{array}{l}\text { Potentiation } \\
(455 \mathrm{~nm})\end{array}$ & $\begin{array}{l}\text { Potentiation } \\
\& \\
\text { Habituation }\end{array}$ & $\begin{array}{c}\sim 206 \% \\
\text { (ionotronic mode) } \\
\sim 165 \% \\
\text { (electronic mode) } \\
\end{array}$ & Yes & Yes & Yes & 10.5 & $\begin{array}{c}\sim 4.8 \\
\text { (ionotronic mode) } \\
\sim 1.3 \times 10^{4} \\
\text { (electronic mode) } \\
\end{array}$ & $\mathrm{SiO}_{2} / \mathrm{Si}$ & {$[23]$} \\
\hline & & $\begin{array}{c}\text { Potentiation } \\
(310 \mathrm{~nm})\end{array}$ & Habituation & $\sim 120 \%$ & No & No & No & $10^{3}$ & $\mathrm{NG}$ & $\mathrm{SiO}_{2} / \mathrm{Si}$ & {$[24]$} \\
\hline & $\mathrm{MoO}_{3}$ & -- & $\begin{array}{c}\text { Potentiation } \\
\& \\
\text { Habituation }\end{array}$ & $\sim 14 \%$ & No & No & No & 1 & 9.6 & $\mathrm{SiO}_{2} / \mathrm{Si}$ & {$[26]$} \\
\hline & $\begin{array}{c}\mathrm{WSe}_{2}, \mathrm{NiPS}_{3}, \\
\mathrm{FePSe}_{3}\end{array}$ & -- & $\begin{array}{c}\text { Potentiation } \\
\& \\
\text { Habituation }\end{array}$ & $\sim 290 \%$ & No & Yes & No & $10^{-1}$ & $3 \times 10^{-2}\left(\mathrm{WSe}_{2}\right)$ & $\mathrm{SiO}_{2} / \mathrm{Si}$ & {$[83]$} \\
\hline & $\mathrm{Cs} \mathrm{PbBr}_{3} \mathrm{QDs}$ & $\begin{array}{c}\text { Potentiation (365- } \\
660 \mathrm{~nm})\end{array}$ & Habituation & $\sim 130 \%$ & No & No & No & $10^{3}$ & $1.4 \times 10^{3}$ & $\mathrm{SiO}_{2} / \mathrm{Si}$ & {$[80]$} \\
\hline & $\begin{array}{c}\text { Graphene/CNT } \\
\text { hybrid } \\
\end{array}$ & $\begin{array}{c}\text { Habituation (405, } \\
532 \mathrm{~nm})\end{array}$ & Potentiation & $\sim 155 \%$ & No & Yes & No & 5 & NG & $\mathrm{SiO}_{2} / \mathrm{Si}$ & {$[21]$} \\
\hline & Graphene & -- & $\begin{array}{c}\text { Potentiation } \\
\& \\
\text { Habituation }\end{array}$ & NG & Yes & No & No & 10 & $5 \times 10^{-1}$ & $\mathrm{SiO}_{2} / \mathrm{Si}$ & {$[20]$} \\
\hline \multirow{5}{*}{ Thin films } & $\mathrm{Ge}_{2} \mathrm{Sb}_{2} \mathrm{Te}_{5}$ & $\begin{array}{l}\text { Potentiation } \\
(1580 \mathrm{~nm})\end{array}$ & -- & No & Yes & No & No & $5 \times 10^{-5}$ & $2.43 \times 10^{2}$ & $\mathrm{Si}_{3} \mathrm{~N}_{4} / \mathrm{SiO}_{2}$ & {$[16]$} \\
\hline & Si nanocrystals & $\begin{array}{c}\text { Potentiation } \\
(375 \mathrm{~nm})\end{array}$ & -- & $\sim 149 \%$ & Yes & No & No & $10^{2}$ & $7 \times 10^{-1}$ & Glass & {$[84]$} \\
\hline & $\mathrm{InGaZnO}_{\mathrm{x}}-\mathrm{Al}_{2} \mathrm{O}_{3}$ & $\begin{array}{l}\text { Potentiation } \\
(365 \mathrm{~nm})\end{array}$ & -- & $\sim 70 \%$ & No & No & No & $10^{2}$ & NG & $\mathrm{Si}$ & {$[28]$} \\
\hline & $\begin{array}{c}\mathrm{InGaZnO}_{\mathrm{x}} \\
\mathrm{InSrZnO}_{\mathrm{x}} \\
\mathrm{InSrO}_{\mathrm{x}}, \mathrm{InZnO}_{\mathrm{x}}\end{array}$ & $\begin{array}{l}\text { Potentiation (385- } \\
\quad 625 \mathrm{~nm})\end{array}$ & -- & Yes & Yes & No & No & 10 & NG & $\mathrm{SiO}_{2} / \mathrm{Si}$ & [29] \\
\hline & $\mathrm{CH}_{3} \mathrm{NH}_{2} \mathrm{PbI}_{3}$ & $\begin{array}{c}\text { Habituation } \\
\text { (along with } \\
\text { electrical pulses) }\end{array}$ & Potentiation & $\sim 40 \%$ & No & No & No & $10^{-1}$ & NG & $\mathrm{SiO}_{2} / \mathrm{Si}$ & [32] \\
\hline
\end{tabular}


